\title{
Not a Grave Finding: Thymic Hyperplasia in the Setting of Graves' Disease
}

\author{
Michael Voss, MD ${ }^{a}$ Zeb ljaz Saeed, MD ${ }^{b}$ Diane Donegan, MB, Bch ${ }^{b}$
}

a Department of Internal Medicine, Indiana University, Ind

b Department of Endocrinology, Diabetes and Metabolism, Indiana University, Ind

\section{Presentation}

A 30-year-old Caucasian male with no previous medical history presented to the emergency department with sudden onset chest pain brought on by exertion at work. He had never experienced similar episodes before, and chest pain was described as severe, mid-sternal, non-radiating, and was associated with palpitations, dyspnea, and near-syncope. Patient also reported unintentional weight loss of 40 pounds over the 3 months leading up to presentation. Review of systems was positive for diaphoresis, increased anxiety, heat intolerance, and a mild hand tremor for several weeks. He denied cough, changes in vision or voice, nausea, vomiting, diarrhea, constipation, or abdominal pain. He reported no history of tobacco or illicit drug use and no family history of thyroid disease or autoimmune conditions.

\section{Assessment}

On examination, the patient was hypertensive (167/94 mm Hg), tachycardic (107 beats per minute), tachypneic (22 breaths per minute) with normal oxygen saturation (98\%) on room air, and afebrile. Salient examination features included mild thyromegaly, resting fine tremor in outstretched hands, and symmetric hyperreflexia. No proptosis, cardiac, or pulmonary abnormalities were noted.

This is the author's manuscript of the work published in final form as:

Voss, M., Saeed, Z. I., \& Donegan, D. (2020). Not a Grave Finding: Thymic Hyperplasia in the Setting of Graves' Disease. The American Journal of Medicine, 133(3), 308-310. 
Electrocardiogram (ECG) demonstrated sinus tachycardia without ischemia, and troponin tests were negative for myocardial injury. Computed tomography (CT) angiography of the chest performed to assess for pulmonary embolism revealed a large $(7.8 \times 3.4 \times 5.1 \mathrm{~cm})$ anterior mediastinal mass, which was solid, without calcification or invasion of adjacent structures, and homogenous in appearance. No pulmonary embolism or mediastinal/hilar lymphadenopathy was seen and lung parenchyma was otherwise normal (Figures $1 \mathrm{~A}$ and $\mathrm{B}$ ). Given the radiological findings, thymic carcinoma, metastatic germ cell tumor, thyroid cancer, and lymphoma formed the initial differential, diagnosis and the patient was admitted for further investigations and biopsy.

In the interim, thyroid function tests were performed given the presenting symptoms, revealing hyperthyroidism with a thyroid stimulating hormone level of $<0.005 \mathrm{mIU} / \mathrm{mL}(0.550-4.780 \mathrm{mIU} / \mathrm{mL})$, free thyroxine level of $3.05 \mathrm{ng} / \mathrm{dL}(0.89-1.76 \mathrm{ng} / \mathrm{dL})$, and total triiodothyronine level of $375 \mathrm{ng} / \mathrm{dL}$ ( 80 200/dL). Endocrinology was consulted for further management and recommended testing confirmed Graves' disease as the cause of hyperthyroidism because his thyroid-stimulating immunoglobulin and his thyroid-hormone receptor antibody titers were both elevated, respectively ( $479 \%$ [Normal < $122 \%]$;

$23.28 \mathrm{IU} / \mathrm{L}[$ Normal $<1.75 \mathrm{IU} / \mathrm{mL}])$. CT-guided biopsy of the mediastinal mass was cancelled because the imaging characteristics in the presence of Graves' disease were most consistent with Graves' diseaseassociated thymic hyperplasia. Close follow-up was recommended.

\section{Management}

Methimazole and propranolol were initiated with subjective improvement in symptoms, and the patient was discharged with close outpatient endocrine follow-up. Clinical course was complicated by methimazole-induced transaminitis, which necessitated discontinuation of thionamide therapy, and definitive therapy with radioactive iodine ablation (RAI) was pursued. The uptake of 24-hour I-123 was increased at $61 \%$ with homogenous gland enlargement. He was treated with $11.95 \mu \mathrm{Ci}$ of oral I-131 
sodium iodide. Treatment was bridged using cholestyramine, prednisone, and propranolol until RAl was effective. Follow-up thyroid hormone levels normalized 1 month after RAI treatment. Chest CT repeated 3 months after RAI (6 months after initial diagnosis) demonstrated significant reduction in the size (2.6 $\times$ $2.5 \times 3.2 \mathrm{~cm}$ ) of the mediastinal thymic mass (Figures $2 \mathrm{~A}$ and B). Subsequent imaging 1 year after RAI therapy was administered demonstrated complete resolution of the thymic mass.

\section{Discussion}

Given this patient's clinical presentation, concerns were initially raised of a cardiac or pulmonary etiology of his symptoms. Appropriate workup including CT imaging demonstrated a large anterior mediastinal mass, which was concerning for a malignancy. However, following the identification of Graves' disease, the correct diagnosis of Graves' disease-associated thymic hyperplasia was made before further unnecessary or invasive interventions.

Graves' disease-associated thymic hyperplasia is a known but often under-recognized finding associated with Graves' disease. Although the exact pathophysiology of Graves' disease-associated thymic hyperplasia is poorly understood, it is believed to be benign.1, 2 It is proposed that thyroid autoimmunity mediated by thyrotropin receptor antibodies and direct hormonal stimulation of thymic cortex in thyrotoxicosis may be the underlying mechanisms.3, 4 CT scan appearance of Graves' diseaseassociated thymic hyperplasia is typically that of a diffusely enlarged thymus gland in the anterior mediastinum with absence of calcifications and no invasion of surrounding structures. However, if suspicious radiographic features are identified, such as a discrete mass within the thymus or internal calcifications, then magnetic resonance imaging (MRI) with chemical shift ratio (CSR) is the next best step. ${ }^{1}$ CSR compares the signal intensity of the thymus to that of a paraspinal muscle. Signal intensity is lower in thymic hyperplasia because of normal fat infiltration in the hyperplastic thymus unlike in thymomas. Thymic biopsy should only be considered if initial CT appearance is suspicious, CSR on MRI is 
worrying or unavailable, or if there is lack of regression of the mass after achieving euthyroid status in Graves' disease. $^{1}$

There are a limited number of case reports in the current literature describing Graves' diseaseassociated thymic hyperplasia. A review by Haider et al described 47 case reports of Graves' diseaseassociated thymic hyperplasia. ${ }^{1}$ Of the 47 cases reviewed, 31 demonstrated similar findings and clinical courses to our patient with significant or complete regression of thymic hyperplasia after treatment of Graves' disease within 6 weeks to 2 years of follow-up. Additionally, included within the review's analysis, is a subset of the 17 case reports that evaluated tissue pathology, which were reassuringly all found to be benign. ${ }^{1}$

In most documented cases of Graves' disease-associated thymic hyperplasia, thymic hyperplasia resolves after initiation of medical therapy or definitive treatment, suggesting it is reasonable to follow patients with serial imaging until resolution.4, 5 Referral for possible invasive interventions should only occur if the mass persists despite resolution of thyrotoxicosis, or if there are additional associated concerning imaging features as detailed previously. Graves' disease-associated thymic hyperplasia is a known complication of Graves' disease and is often an incidental finding. With the increased use of imaging modalities in medicine, the frequency of these incidental discoveries will likely continue to increase. Therefore, clinicians need to be aware of the association between benign thymic hyperplasia and Graves' disease to avoid unnecessary invasive interventions, counsel the patient appropriately, and provide adequate follow-up. 


\section{References}

1. Haider U, Richards P, Gianoukakis AG. Thymic Hyperplasia associated with graves' disease: pathophysiology and proposed management algorithm. Thyroid 2017;27(8):994-1000.

2. Sherer $\mathrm{Y}$, Bardayan $\mathrm{Y}$, Shoenfeld $\mathrm{Y}$. Thymoma, thymic hyperplasia, thymectomy and autoimmune diseases (Review). Int J Oncol 1997;10 (5):939-43.

3. Song YS, Won JK, Kim MJ, et al. Graves' patient with thymic expression of thyrotropin receptors and dynamic changes in thymic hyperplasia proportional to Graves' disease activity. Yonsei Med J $2016 ; 57(3): 795-8$.

4. Dalla Costa M, Mangano FA, Betterle C. Thymic hyperplasia in patients with Graves' disease. J Endocrinol Investig 2014;37(12):1175-9.

5. Desforges-Bullet V, Petit-Aubert G, Collet-Gaudillat C, et al. Thymic hyperplasia and Graves' disease: a non-fortuitous association. Case report and review of literature. Ann Endocrinol (Paris) 2011;72 (4):304-9. 


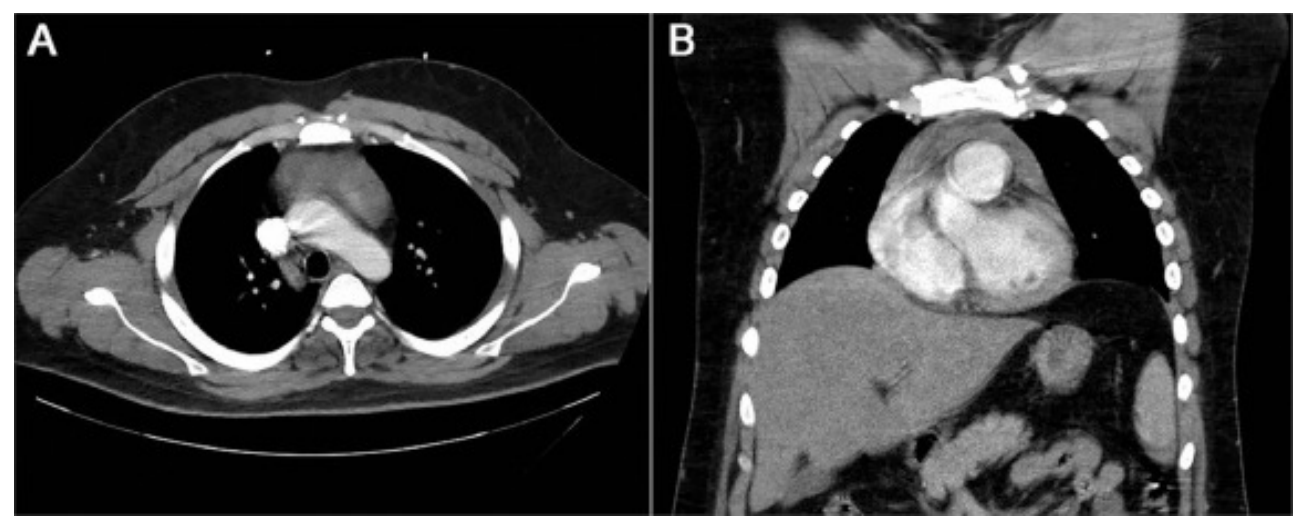

Figure 1. Images from chest computed tomography (CT) with intravenous contrast (pulmonary embolism protocol) with both axial (A) and coronal (B) views of the thymic mass at time of patient's initial presentation. Overall thymic dimensions measured $7.8 \times 3.4 \times 5.1 \mathrm{~cm}$.

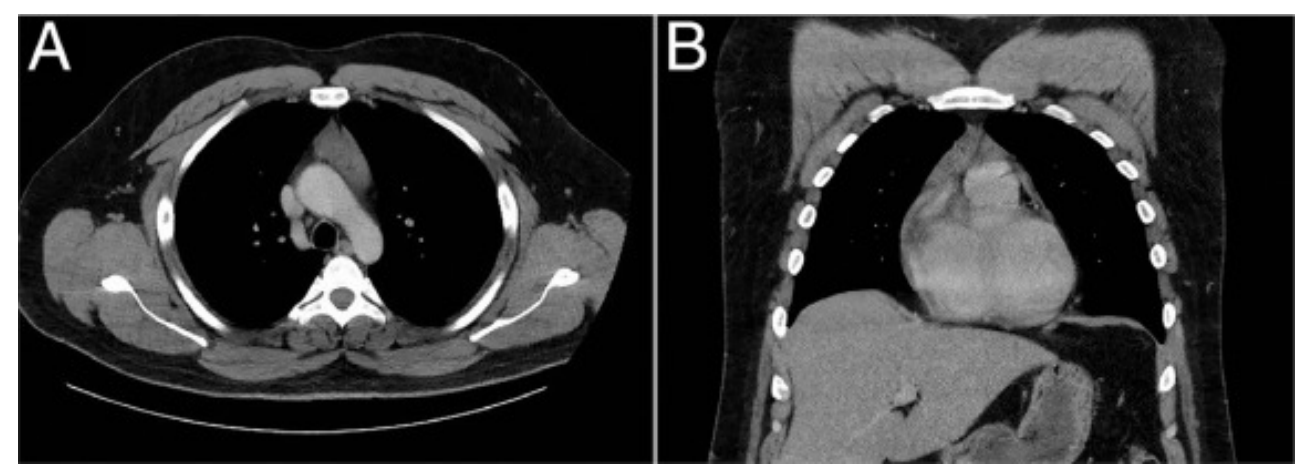

Figure 2. Images from follow-up chest computed tomography (CT) without intravenous contrast obtained 3 months after radioactive iodine ablation (RAI) therapy ( 6 months after initial diagnosis) with both axial (A) and coronal (B) views. Thymic dimensions measured $2.6 \times 2.5 \times 3.2 \mathrm{~cm}$. 